\title{
Gene therapy hits a nerve
}

Poor blood flow in the lower limbs is a significant health problem that leads to neurological symptoms such as chronic pain, sensory impairment reflex loss and muscle wasting and weakness. These result from pathological alterations in peripheral nerves, including loss of myelin and axonal degeneration. Despite advances in surgical and percuteaneous revascularization techniques, the prognosis remains poor. In the current issue of Nat. Med. (6, 405-413) Jeffrey Isner and colleagues have investigated whether neurological symptoms can be prevented/reversed by intra-

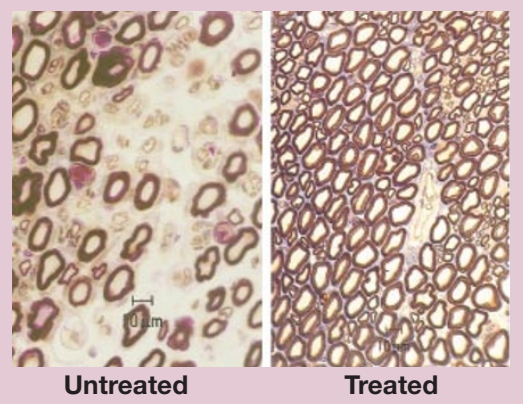
muscular transfer of a gene encoding an endothelial cell mitogen used to promote therapetic angiogenesis. Using a well-established animal model of hindlimb ischemia, the authors transferred naked DNA encoding vascular endothelial factor (VEGF) 10 days after the induction of ischemia and studied neuronal activity. Sure enough, nerve function was restored earlier and /or recovered faster in treated animals. These findings were in part due to enhanced limb perfusion, but also indicated a direct effect of VEGF on neural integrity. Indeed, their results suggest that VEGF may act directly on Schwann cells during nerve recovery by protecting them against hypoxia-induced apoptosis arising from the peripheral ischemia.

\section{Reversible immortality}

A multinational team of researchers has shown that acute liver failure in a rat model can be prevented using a reversibly immortalized line of human hepatocytes. Though transplantation of primary hepatocytes has been suggested as a treatment for liver failure, the new work (Science 287, 1258-1262, $2000)$ is the first to demonstrate that liver cells can be reversibly immortalized for growth in culture, potentially overcoming the shortage of transplantable liver cells that limits this type of treatment. The team immortalized a differentiated hepatocyte line by inserting the SV40 $\mathrm{T}$ antigen gene flanked by LoxP recombination target sites. Once the cell population had expanded in culture, the SV40 gene was removed by expressing Cre recombinase, an enzyme that excises LoxP-flanked sequences, in the cells. Removing the SV40 gene aimed to lower the possibility of the cells causing tumors after transplantation. Transplanting the hepatocytes rescued rats that had undergone removal of $90 \%$ of the liver, a normally fatal operation that simulates acute liver failure. Philippe Leboulch, a senior author on the paper, says that the approach might eventually be used as "short-term therapy to bridge patients who have an acute liver failure...while they're waiting for a liver transplant."

Research News Briefs written by Alan Dove and Robert Frederickson.

\section{Bionic shocker}

A cleverly designed silicon chip developed by researchers at the University of California (Berkeley, CA) may soon give biologists precise control over cell electroporation. Using standard silicon fabrication techniques, the researchers created a chip with a microscopic hole capable of holding a single mammalian cell as part of an electrical circuit. The cell behaves as a diode in the circuit, allowing current to flow at the specific voltage that causes pores to open in the cell membrane. The ability to monitor electroporation in real time in a single cell should allow scientists to exert precise control on the introduction of novel genes, proteins, and other macromolecules. Since individual cells in a population differ in their responses to electricity, current electroporation techniques are often inefficient. Though the initial work, reported in Biomedical Microdevices (3, 145-150, 2000), involved manually drawing each cell into the hole in the chip, a commercially useful device might electroporate cells in a continuously flowing stream. In addition, it could be "the first step to building complex circuitry that incorporates the living cell," says Boris Rubinsky, senior author on the paper. Cells implanted on chips could ultimately be used to administer precise doses of therapeutic proteins, build complex biocomputers, or test novel pharmaceutical compounds in automated high-throughput screening systems.

\section{Particles on the nose}

Scientists at the Massachusetts Institute of Technology (Cambridge, MA) have developed a new technique for creating plastic foams that could be used in a wide array of medical and industrial applications (Proc. Natl. Acad. Sci. USA 97, 1970-1975, 2000). Their approach uses uniformly sized hydrocarbon particles as a template that can subsequently be removed by a solvent, leaving behind an intact polymer foam. The size and chemistry of the wax particles determines the size of the pores in the foam and the final structure of the material. To test the system, the team carved a block of foam made with a biodegradable polymer into the shape of a human nose, then seeded the block with bovine chondrocytes. After a twoweek incubation in a bioreactor, sections of the artificial nose showed a stable composite made of cartilaginous tissue and biodegradable polymer. Another experiment demonstrated that an enzyme could be chemically linked to the biodegradable foam, allowing it to be slowly released in active form as the polymer breaks down. Apart from medical applications, the technique could be used to design catalyst beds and enzyme-immobilized bioreactors.

\section{Amyloid inhibitor get rational}

A structure-based rational drug design approach has produced new compounds capable of inhibiting the formation of insoluble protein fibrils found in certain genetic diseases. The research focused on transthyretin (TTR), a protein that can form amyloid fibers that damage neural or cardiac tissue. Though TTR-associated amyloid diseases are thought to be relatively rare, the insoluble structures are biophysically similar to the fibrils formed in Alzheimer's disease. In the new work, the researchers used $\mathrm{x}$-ray crystallography to develop detailed three-dimensional structures of TTR bound to compounds that have been shown to inhibit TTR amyloidosis. Using this structural information, the team then synthesized new small molecules that they predicted would act more specifically on TTR, without the side effects of current drug treatments. Subsequent co-crystallization of TTR bound to the newly synthesized compounds verified the theoretical predictions. "We're...trying to use a similar approach for Alzheimer's disease, but that of course requires structural information, and that's what everyone is trying to get right now," says senior author James Sacchetini. The findings are reported in Nature Structural Biology $(7,312-321,2000)$ and the J. Am. Chem. Soc. 122, 2178-2192, 2000) 